\title{
Entwicklung der Verbstellung beim fortgeschrittenen Erwerb des Deutschen als Fremdsprache
}

\author{
Development of verb placement in acquisition \\ of German as a foreign language
}

\begin{abstract}
This article investigates the acquisition of verb placement in German as a foreign language at an advanced stage of development. The main objective of the investigation is to analyse written and spoken language production in terms of the use of subject-verb-inversion, verbal bracket, and verb-final placement in subordinate clauses. The results reveal a discrepancy between written and spoken language production with respect to correct usage of the verb placement rules. While correctness in the written production task exceeded $97 \%$ for all phenomena, the oral translation task generated less correct sentences, but only for inversion and verb-final placement. The non-target usage of inversion and verb-final pattern in spoken production points to processing problems when translating from Polish into German, which are further confirmed by lower accuracy for these two phenomena. At the moment of testing, the verbal bracket has already been acquired, which is in line with the universal developmental sequence in the acquisition of German syntax.
\end{abstract}

KEYWORDS: verb placement, German as a foreign language, foreign language learning, interlanguage grammar.

SCHLÜSSELWÖRTER: Verbstellung, Deutsch als Fremdsprache, Fremdsprachenerwerb, Interimsgrammatik.

\section{EINFÜHRUNG}

In der Erforschung des unterrichtlichen Fremdsprachenerwerbs sieht die Sprachdidaktik eine besondere Aufgabe, ohne die eine Verbesserung sowie eine Optimierung der Fremdsprachenvermittlung kaum möglich ist. Im Rahmen der Sprachlehrforschung unterstreichen Edmondson und House (2006: 4), dass es sinnlos sei, „nach didaktischen Prinzipien zu suchen, ohne eine durchdachte Theorie des Fremdsprachenlernens erarbeitet zu haben". Um Prinzipien des Lehrverhaltens festlegen zu können, muss demnach gleichzeitig oder vorher 
deren Auswirkung auf Lerner erforscht werden. Eine eingehende Beschäftigung mit der menschlichen Fähigkeit zum Lernen von Fremdsprachen wird auch in der glottodidaktischen Forschung in Polen postuliert (vgl. Pfeiffer 2001; Sadownik 1997; Sopata 2018). Dazu sollten fremdsprachliche Lern- und Lehrprozesse beobachtet, beschrieben und analysiert werden. Für eine empirisch begründete Optimierung der Fremdsprachenvermittlung sind „Erkenntnisse der Linguistik über die Mechanismen der menschlichen Sprachbeherrschung und der sie strukturierenden Prinzipien von besonderer Wichtigkeit“" (Sopata 2004: 149).

Es besteht gegenwärtig kein Zweifel mehr daran, dass auch der institutionelle Fremdsprachenerwerb ein systematischer, nach inneren Gesetzmäßigkeiten ablaufender Prozess ist, der sich von außen nur bedingt steuern lässt. Dies betrifft insbesondere den Grammatikerwerb, dem in der Forschung zum Deutschen als Fremdsprache bereits viel Beachtung geschenkt wurde. Die Bemühungen um eine systematische und umfassende Erwerbstheorie brachten eine Reihe von gesicherten Erkenntnissen hervor, die den universalen Charakter des gesteuerten Grammatikerwerbs des Deutschen unter Beweis stellten (vgl. Kapitel 2).

Die vorliegende Studie schließt sich diesen Bemühungen an, indem sie der Verbstellung polnischer DaF-Lerner ${ }^{1}$ in schriftlicher und mündlicher Sprachproduktion auf den Grund geht. Insbesondere soll hierbei die Frage geklärt werden, welche Schwierigkeiten beim Gebrauch der grundlegenden Verbstellungsmuster in einem fortgeschrittenen Erwerbsstadium festgestellt werden können. Daraufhin werden die festgestellten Fehler im Zusammenhang mit einer natürlichen Erwerbsequenz und syntaktischem Transfer aus den bereits erworbenen Sprachen untersucht.

\section{ZUM ERWERB DER VERBSTELLUNG IM DEUTSCHEN ALS FREMDSPRACHE}

Die Verbstellung in der deutschen Sprache gehört ohne Zweifel zu den meistuntersuchten Phänomenen in der Spracherwerbsforschung. Zahlreiche Studien, sowohl zum Erstspracherwerb als auch zum Zweitspracherwerb von Kindern und Erwachsenen, deuten darauf hin, dass die Entwicklung der Verbstellungsregularitäten in einer geordneten Entwicklungssequenz abläuft. Diese ist weitgehend universell und bleibt - unabhängig von den Erstsprachen der untersuchten Populationen - unverändert.

\footnotetext{
${ }^{1}$ Aus Gründen der Lesbarkeit wird in diesem Beitrag bei Personenbezeichnungen die maskuline Sprachform verwendet. Es sind stets Personen aller Geschlechter gleichermaßen gemeint.
} 
Intensive Untersuchungen zum Verlauf des Wortstellungserwerbs im natürlichen Zweitspracherwerb Erwachsener wurden vor allem im Rahmen des bekannten ZISA-Projekts durchgeführt (Meisel, Clahsen \& Pienemann 1981; Clahsen, Meisel \& Pienemann 1983). Mittels informeller Interviews wurden insgesamt 45 erwachsene Gastarbeiter untersucht, die Deutsch als ihre Zweitsprache erworben haben. Die erhobenen Querschnittsdaten ermöglichten es, folgende sechs Entwicklungsstadien zu spezifizieren (vgl. Clahsen \& Muysken 1986: 106-107):

1. Phase - SVO: Keine Wortstellungsregel ist implementiert. Konstituenten erscheinen in der festen linearen Abfolge: NP (AUX / MOD) V (NP) (PP).

2. Phase - ADV-PREP: Adverbien und Präpositionalphrasen werden fakultativ an die satzinitiale Position verschoben, ohne die SV-Abfolge zu beeinflussen. Infolgedessen werden inkorrekte V3-Strukturen produziert.

3. Phase-PARTIKEL: Infinite Verbteile, d.h. Verbpartikeln, Infinitive und Partizipien werden an die satzfinale Position verschoben.

4. Phase - INVERSION: Bei Voranstellung einer Phrase treten finite Verben vor das Subjekt, sodass eine XVS-Abfolge entsteht, die in der zweiten Phase fehlt.

5. Phase - ADV-VP: Adverbien können optional zwischen dem finiten Verb und dem Objekt erscheinen.

6. Phase - V-ENDE: Finite Verben besetzen in Nebensätzen die Verbendstellung.

Die Phasen des natürlichen Zweitspracherwerbs konnten interessanterweise auch beim institutionellen Zweitspracherwerb festgestellt werden. Von besonderer Bedeutung für die vorliegende Studie ist die Untersuchung von Barczyk (2008), die schriftliche Sprachproduktion polnischer Deutschlerner im Alter zwischen 20 und 22 Jahren untersuchte. Sie analysierte persönliche und halbformelle Briefe, die in ca. zwei- bis dreiwöchigen Intervallen verfasst wurden. Innerhalb von 19 Monaten haben die Probanden insgesamt 15 Texte produziert, die dann auf die Realisierung der Inversion, Verbalklammer und Verbendstellung hin ausgewertet wurden. Die Ergebnisse der Analyse zeigten, dass die Regel der Verbalklammer kein Erwerbsproblem für die Probanden war. Bereits zu Beginn der Untersuchung wurde die Distanzstellung grundsätzlich korrekt realisiert und die Richtigkeitsrate betrug durchgehend nicht weniger als 75 Prozent.

Demgegenüber verlief die Entwicklung der Inversion und der Verbendstellung in Nebensätzen alles andere als reibungslos. Barczyk (2008: 381) stellte dabei große Schwankungen in der Entwicklung dieser Phänomene fest. Außerdem waren die Lerner nicht imstande, die Verbendstellung auf alle eingeleiteten Nebensätze anzuwenden. Stattdessen erlernten sie sie Item für Item, was die Autorin als Indiz dafür deutete, dass die natürlichen Erwerbsmechanismen im Fremdsprachenunterricht durch das Strukturlernen ersetzt werden können. 
Die meisten Schwierigkeiten hatten die Lerner mit den Nebensatzeinleitern "weil / da" und "wenn“ sowie mit Fragepronomen. Am wenigsten Probleme bereiteten dagegen die nebensatzeinleitenden Konjunktionen "obwohl“ und "dass" sowie Relativpronomen. Barczyk (2008) deckte in ihren Untersuchungen folgende Erwerbsfolge auf:

1. SVO - kanonische Wortfolge

2. Verbalklammer

3. Subjekt-Verb-Inversion

4. Verbendstellung

Diese für den DaF-Erwerb ermittelte Entwicklungssequenz stimmt folglich mit dem natürlichen DaZ-Erwerb Erwachsener weitestgehend überein, auch wenn den zwei Erwerbstypen unterschiedliche Mechanismen zugrunde liegen können.

Es wurden viele Versuche unternommen, die Hypothese der natürlichen Erwerbssequenz bei Lernern verschiedener Erstsprachen zu überprüfen. Ellis (1989) untersuchte beispielsweise die Phasen des Verbstellungserwerbs bei 39 erwachsenen Deutschlernern mit Englisch als Erstsprache, indem er anhand einer Bildbeschreibungsaufgabe elizitierte Daten einer Analyse unterzog. Seine Untersuchung zielte einerseits auf einen Vergleich von den in der ZISA-Studie ermittelten Erwerbsphasen mit denen im gesteuerten Erwerb des Deutschen ab. Dabei sollte nachgewiesen werden, dass beide Erwerbsverläufe ähnlich sind. Andererseits wollte er die Frage beantworten, ob die explizite Instruktion einen Einfluss auf den Verlauf des Verbstellungserwerbs hat. Ellis (1989: 321) kam zu folgendem Schluss:

The acquisition of syntactical features, such as German word order rules, follows the same sequence in both untutored and tutored learners. The classroom learners followed this sequence even though the order of introduction and emphasis given to the rules in the instruction was different (Ellis 1989: 321).

Interessanterweise durchliefen die untersuchten Deutschlerner die Erwerbsphasen schneller als die Probanden in der ZISA-Studie, was als Vorteil des gesteuerten Zweitspracherwerbs gegenüber dem ungesteuerten Zweitspracherwerb hinsichtlich des Erwerbstempos interpretiert werden kann. Beispielsweise haben sich fast alle schon nach 22 Kontaktmonaten die Distanzstellung angeeignet.

Zu den meistzitierten Studien zum DaF-Erwerb der Grammatik gehört zweifelsohne das DiGS-Projekt (Projekt Deutsch in Genfer Schulen), bei dem die grammatische Entwicklung bei frankophonen Lernern ins Visier genommen wurde (vgl. Diehl, Christen, Leuenberger, Pelvat \& Studer 2000). Das Projekt zielte darauf ab, die Hypothese der kognitiv orientierten Zweitspracherwerbs- 
forschung zu überprüfen. Diese besagt, dass der Erwerb einer Fremdsprache auch im institutionellen Kontext inneren Gesetzmäßigkeiten unterliegt und in einer geordneten Phasenabfolge verläuft, die durch externe Instruktionsmaßnahmen nicht verändert werden kann. Die von Diehl et al. (2000) vorgeschlagene Erwerbsreihenfolge weist zwar viele Parallelen zu den oben erwähnten Studien auf, sie unterscheidet sich aber von diesen in einem wesentlichen Aspekt: Die Inversion wird laut den Autoren sehr spät, und zwar erst nach der Aneignung der Nebensätze, erworben.

In einer Folgestudie untersuchte Terrasi-Haufe (2004) anhand mündlicher und schriftlicher Daten den DaF-Erwerb von Lernern an Tessiner Schulen. Die Analysen des erstellten Korpus deuteten auf eine ähnliche Erwerbssequenz hin, in der die Inversion nach topikalisierten Adverbialen und einleitenden Konjunktionaladverbien als Letzte erworben wurde. Auch Tschirner (1999) wies aufgrund mündlicher Daten auf persistente Schwierigkeiten mit der Inversion und der Nebensatzstellung im DaF-Erwerb hin. Bei seinen erwachsenen Deutschlernern mit englischer Erstsprache lag der Prozentsatz korrekt angewendeter Regeln nach 225 Stunden Instruktion bei 92 Prozent für Satzklammer, 34 Prozent für Inversion und nur 24 Prozent für Nebensatzstellung. Weitere Befunde, die auf die in der ZISA-Studie ermittelte, natürliche Erwerbsfolge hinweisen, sind auch Ballestracci (2007) und Sadownik (2008) zu entnehmen.

Zusammenfassend zeigen die besprochenen Studien, dass der Erwerb der Inversion und der Verbendstellung in Nebensätzen eine große Herausforderung für Deutschlerner darstellt, die durch explizite Instruktionsmaßnahmen im Unterricht kaum bewältigt werden kann. Ferner setzt sich die natürliche Erwerbssequenz, unabhängig von den Erstsprachen der Probanden und den angewandten Untersuchungsmethoden, durch.

\section{ALLGEMEINES ZUR VERBSTELLUNG IM DEUTSCHEN UND IM POLNISCHEN²}

Deutsch und Polnisch unterscheiden sich im Hinblick auf die drei untersuchten Phänomene deutlich voneinander, wodurch sie eine Steilvorlage für komparative Analysen aus spracherwerbstheoretischer Sicht bieten. Der Hauptunterschied besteht wohl darin, dass die für das Deutsche charakteristische Verbzweitstellung, die auch mit der Subjekt-Verb-Inversion verbunden ist, keine obligatorische Eigenschaft der polnischen Sprache ist:

${ }^{2}$ Dieses Kapitel wurde in seinen wesentlichen Teilen aus dem Artikel von Sopata und Długosz (2020) übernommen. 
1. Ania idzie dzisiaj do szkoty. Ania geht heute zur Schule.

2. Dzisiaj Ania idzie do szkoty. *Heute Ania geht zur Schule.

Den obigen Beispielen ist zu entnehmen, dass das finite Verb im Polnischen sowohl in V2 als auch in V3 stehen kann, ohne dass der Satz ungrammatisch ist.

Der nächste Unterschied betrifft topologische Regularitäten, die im Deutschen viel ausgeprägter sind. In der polnischen Sprache ist der Verbalkomplex im Gegensatz zur deutschen Sprache prinzipiell einteilig und daher nicht klammerbildend (vgl. Engel et al. 2000: 495). Es ist aber nicht unmöglich, dass ein Satzglied, z. B. ein Objekt, zwischen den beiden Teilen des Verbalkomplexes erscheint:

3. Ania musi posprzatać swój pokój.

*Ania muss aufräumen ihr Zimmer.

4. Ania musi swój pokój posprzatać.

Ania muss ihr Zimmer aufräumen.

Wenngleich Satz 4 grammatikalisch korrekt ist, erscheint er einem polnischen Muttersprachler befremdlich. Permutationen der Wortstellung sind zwar zugelassen, aber nur in Abhängigkeit von pragmatischen und informationsstrukturellen Faktoren. Das Polnische weist demnach grundsätzlich Kontaktstellung, das Deutsche hingegen Distanzstellung auf.

Ein wesentliches Charakteristikum des Deutschen ist ferner die Verbendstellung in Nebensätzen. Im Polnischen ist diese nicht existent und die Verbposition in Nebensätzen entspricht der in Hauptsätzen:

5. Jan wie, że Ania idzie do kina.

*Jan weiß, dass Ania geht ins Kino.

'Jan weiß, dass Ania ins Kino geht'

Insgesamt zeigt sich, dass die drei untersuchten Phänomene in beiden Sprachen unterschiedlich realisiert werden. Das kommt vor allem daher, dass die Verbposition im Deutschen im Vergleich zum Polnischen fest verankert ist.

\section{STUDIE}

\subsection{Forschungsfragen und Hypothesen}

Das Hauptziel der vorliegenden Studie ist die Untersuchung der Verbstellung in der Interimsgrammatik polnischer DaF-Lerner in einem fortgeschrittenen Er- 
werbsstadium. Anvisiert wird die Realisierung folgender drei Verbstellungsmuster: (1) Inversion, (2) Verbalklammer und (3) Verbendstellung in Nebensätzen. Eine wesentliche Stärke der Studie besteht darin, dass sie gleichzeitig schriftliche und mündliche Sprachproduktion betrachtet. Zudem wurden polnische DaFLerner mit sehr guten Englischkenntnissen als Probanden gewählt, wodurch die Ergebnisse potenziell im Zusammenhang mit dem Transfer aus der ersten Fremdsprache ausgewertet werden können. ${ }^{3}$ Vor dem Hintergrund der oben skizzierten Untersuchungen zum Erwerb der Verbstellungsregularitäten im Deutschen als Fremdsprache und der allgemeinen Ziele der Studie werden hier folgende Forschungsfragen gestellt:

1. Wie werden die drei untersuchten Verbstellungsmuster in einem fortgeschrittenen Erwerbsstadium angewendet?

2. Stimmt die Entwicklung der drei untersuchten Phänomene mit der natürlichen Erwerbssequenz überein?

3. Lassen sich Unterschiede zwischen der schriftlichen und der mündlichen Sprachproduktion im Hinblick auf die drei untersuchten Phänomene nachweisen?

4. Können abweichende Verbstellungsmuster mit dem Transfer aus der ersten Fremdsprache in Verbindung gebracht werden?

Da sich die Probanden bereits in einem fortgeschrittenen Erwerbsstadium befinden, wird erwartet, dass sie keine Schwierigkeiten mit der Distanzstellung haben, welche relativ früh erworben wird. Problematisch für sie könnten dagegen die Inversion und die Verbendstellung sein, die gewöhnlich sogar bei weit fortgeschrittenen Lernern als Fehlerquelle diagnostiziert werden. Dies würde dann im Einklang mit der natürlichen Erwerbssequenz stehen und zugleich die Möglichkeit des Transfers aus dem Englischen deutlich einschränken. Im Hinblick auf die dritte Forschungsfrage werden wesentlich weniger Fehler in der schriftlichen Sprachproduktion erwartet, weil beim Schreiben mehr Planungszeit zur Verfügung steht, wodurch die Lerner sowohl auf das explizite Regelwissen wie auch auf das metasprachliche Wissen zugreifen können.

\subsection{Probandencharakteristik}

An der Studie nahmen zehn polnische Studierende des Bachelorstudiengangs Angewandte Linguistik freiwillig teil. Vor dem Studienbeginn hatten alle in der Schule Deutsch- und Englischunterricht. Ihre aktuelle Inputsituation ist sehr ver-

\footnotetext{
${ }^{3}$ Zwischensprachliche Einflüsse beim Erwerb des Deutschen als Tertiärsprache bei polnischen Lernern werden u. a. von Zuzok (2010) und Długosz (2018) thematisiert.
} 
gleichbar, weil sie von denselben Dozierenden unterrichtet werden und dieselben Lernmaterialien benutzen. Alle unterhalten sich gelegentlich mit deutschen und englischen Muttersprachlern und haben kurze Auslandsaufenthalte hinter sich. Tabelle 1 fasst die wichtigsten Informationen über die Probanden zusammen.

Tabelle 1. Übersicht über die wichtigsten Informationen über die Probanden

\begin{tabular}{|l|c|c|c|c|c|}
\hline Proband(in) & Alter & $\begin{array}{c}\text { Angegebenes } \\
\text { Sprachniveau } \\
\text { im Deutschen }\end{array}$ & $\begin{array}{c}\text { Angegebenes } \\
\text { Sprachniveau } \\
\text { im Englischen }\end{array}$ & $\begin{array}{c}\text { Kontaktdauer } \\
\text { mit Deutsch } \\
\text { in Jahren }\end{array}$ & $\begin{array}{c}\text { Kontaktdauer } \\
\text { mit Englisch } \\
\text { in Jahren }\end{array}$ \\
\hline Lena & 21 & B2 & B1 & 8 & 15 \\
\hline Ala & 25 & B1 & B2 & 15 & 21 \\
\hline Maks & 20 & C1 & B2 & 12 & 7 \\
\hline Kaja & 20 & B1 & B2 & 10 & 14 \\
\hline Ola & 23 & C1 & C1 & 2 & 6 \\
\hline Maja & 21 & B1 & B2 & 10 & 10 \\
\hline Anna & 21 & C1 & C1 & 14 & 16 \\
\hline Nina & 20 & B2 & B1 & 9 & 6 \\
\hline Julia & 21 & B2 & C1 & 4 & 14 \\
\hline Pola & 21 & B2 & C1 & 4 & 10 \\
\hline
\end{tabular}

Aus der Tabelle kann man ablesen, dass die Probanden in Bezug auf das Sprachniveau in beiden Sprachen eine eher heterogene Gruppe bilden. Bei sieben von ihnen kann Deutsch, nach dem Kriterium der Erwerbsreihenfolge, als Tertiärsprache klassifiziert werden, weil sie nach Englisch gelernt wurde. Nur drei Personen gaben an, dass Deutsch ihre dominante Sprache ist. Die übrigen Probanden schätzten ihr Niveau im Englischen höher ein als im Deutschen. Die Probandencharakteristik ermöglicht es also, den eventuellen Einfluss der stärkeren zweiten Fremdsprache auf die Sprachproduktion in der schwächeren dritten Fremdsprache im syntaktischen Bereich aufzuspüren.

\subsection{Untersuchungsdesign}

Die Studie bestand aus zwei Aufgaben, die Antworten auf die gestellten Forschungsfragen liefern sollten. Um den Gebrauch der anvisierten Verbstellungsmuster in der schriftlichen Sprachproduktion zu untersuchen, wurden die Probanden zuerst gebeten, einen Aufsatz im Umfang von 200 bis 250 Wörtern zu schreiben. Sie hatten zwei Themen zur Wahl, die zuvor im Unterricht behandelt worden waren. Die Zeit zur Ausführung der Aufgabe war unbegrenzt. 
Die Probanden durften dabei keine Wörterbücher benutzen. Die zweite Aufgabe wurde als Übersetzungsaufgabe konzipiert, bei der schriftlich dargebotene Sätze in polnischer Sprache mündlich ins Deutsche übersetzt werden sollten. ${ }^{4}$ Auf dem Bildschirm eines Laptops erschien für sieben Sekunden ein Satz in polnischer Sprache und die Probanden wurden gebeten, den Satz möglichst schnell mündlich ins Deutsche zu übersetzen. Die Zeit war begrenzt, aber die Probanden hatten die Möglichkeit, mit einem Tastendruck zu einem nächsten Satz überzugehen. Zur Gestaltung und Durchführung der Übersetzungsaufgabe sowie zur Antworterfassung wurde die E-Prime 2.0 Professional SP2-Software angewendet.

\subsection{Material}

Die Übersetzungsaufgabe umfasste insgesamt 26 Sätze, anhand derer Äußerungen mit den untersuchten Verbstellungsmustern elizitiert werden sollten. Eine Übersicht über die Sätze liefert Tabelle 2.

Tabelle 2. Übersicht über die Sätze in der Übersetzungsaufgabe

\begin{tabular}{|l|c|c|c|}
\hline \multicolumn{1}{|c|}{ Phänomen } & Inversion & Verbalklammer & $\begin{array}{c}\text { Verbendstellung } \\
\text { in Nebensätzen }\end{array}$ \\
\hline Zahl der Sätze & 12 & 9 & 5 \\
\hline
\end{tabular}

Die Sätze zur Elizitierung der Inversion begannen mit einem Adverb (acht Sätze) oder einer Präpositionalphrase (zwei Sätze), z. B. Potem spotykam się z kolegami (Dann treffe ich mich mit meinen Freunden). Zwei weitere Sätze wurden mit einem Nebensatz eingeleitet, sodass das Verb im übergeordneten Satz vorangestellt werden musste, z. B. Kiedy stucham radia, zazwyczaj czuję się zrelaksowany (Wenn ich Radio höre, fühle ich mich in der Regel entspannt). Neun Sätze enthielten mehr als eine Angabe im Mittelfeld, z. B. Dlatego chyba chciałabym studiować w przyszłości za granica (Deswegen möchte ich in Zukunft vielleicht im Ausland studieren).

Die Sätze zur Elizitierung der Verbalklammer enthielten in den meisten Fällen Modalverben (sieben Sätze), z. B. Moge przyjść do ciebie za godzinę (Ich kann in einer Stunde zu dir kommen). Ein Satz enthielt eine konjugierte Form des Verbs „zapominać" („vergessen“) in Kombination mit dem Infinitiv "mówićc

${ }^{4}$ Für diese Art Übersetzung finden sich in der deutschsprachigen Literatur die Bezeichnungen „Blattdolmetschen“, „Vom-Blatt-Übersetzen“ oder „Stegreifübersetzen“. Da beim Blattdolmetschen ein schriftlicher Quelltext mündlich übertragen wird, besteht Uneinigkeit darüber, ob man in diesem Fall vom Dolmetschen oder eher vom Übersetzen sprechen sollte (vgl. Odendahl 2016). Diese Nomenklatur-Diskussion ist allerdings nicht relevant für die vorliegende Studie. 
(„sprechen“), sodass in der deutschen Übersetzung eine Infinitivkonstruktion mit „zu“ notwendig war. Ein Satz drückte die Vergangenheit aus, um die Probanden dazu bewegen, einen deutschen Satz im Perfekt zu bilden.

Das letzte Phänomen, also die Verbendstellung in Nebensätzen, wurde anhand von fünf Sätzen elizitiert. Drei Sätze enthielten nebensatzeinleitende Konjunktionen wie „że“ ("dass"), "ponieważ“ („weil") und „żeby" (,damit"), z. B. Powiedzieliście im, że dzisiaj piszemy test? (Habt ihr ihnen gesagt, dass wir heute einen Test schreiben?). Ein Nebensatz enthielt das Fragepronomen "jak“ („wie“) und musste dementsprechend mit der Verbendstellung ins Deutsche übersetzt werden. Ein Satz war ein temporaler Nebensatz mit "kiedy“ („wenn“). Zwölf Sätze enthielten zusätzlich Kognaten, also Wörter, die in zwei oder mehreren Sprachen parallel vorkommen und semantische, morphologische sowie phonologische Eigenschaften gemeinsam haben, z. B. „Park“ (vgl. Dijkstra 2009: 252-253). Das Ziel war dabei, herauszufinden, ob die Kognaten einen syntaktischen Transfer oder ein Code-Switching induzieren können.

\subsection{Ergebnisse der Aufgabe zur schriftlichen Produktion}

Die Analyse der von den Probanden verfassten Aufsätze wurde auf das Vorkommen der drei untersuchten Verbstellungsmuster hin durchgeführt. Zuerst wurden alle Sätze aus den Texten extrahiert, die die Inversion, Verbalklammer und Verbendstellung in Nebensätzen enthielten (Kontexte). In einem nächsten Schritt wurde überprüft, in wie vielen Sätzen sie inkorrekt realisiert wurden (Fehler). Dann wurde die durchschnittliche Korrektheit für jedes Verbstellungsmuster separat berechnet. Tabelle 3 stellt die Ergebnisse der Analyse zusammen.

Tabelle 3. Ergebnisse der Aufgabe zur schriftlichen Sprachproduktion

\begin{tabular}{|l|c|c|c|c|c|c|}
\hline \multirow{2}{*}{ Proband(in) } & \multicolumn{2}{|c|}{ Inversion } & \multicolumn{2}{c|}{ Verbalklammer } & \multicolumn{2}{c|}{$\begin{array}{c}\text { Verbendstellung } \\
\text { in Nebensätzen }\end{array}$} \\
\cline { 2 - 7 } & Kontexte & Fehler & Kontexte & Fehler & Kontexte & Fehler \\
\hline Lena & 6 & 1 & 3 & 0 & 9 & 0 \\
\hline Ala & 6 & 1 & 7 & 1 & 8 & 1 \\
\hline Maks & 6 & 0 & 4 & 0 & 12 & 0 \\
\hline Kaja & 11 & 0 & 6 & 1 & 12 & 0 \\
\hline Ola & 7 & 0 & 6 & 0 & 7 & 0 \\
\hline Maja & 6 & 0 & 10 & 0 & 11 & 0 \\
\hline Anna & 12 & 0 & 8 & 0 & 9 & 0 \\
\hline Nina & 9 & 0 & 11 & 0 & 10 & 0 \\
\hline
\end{tabular}




\begin{tabular}{|l|c|c|c|c|c|c|}
\hline \multirow{2}{*}{ Proband(in) } & \multicolumn{2}{|c|}{ Inversion } & \multicolumn{2}{c|}{ Verbalklammer } & \multicolumn{2}{c|}{$\begin{array}{c}\text { Verbendstellung } \\
\text { in Nebensätzen }\end{array}$} \\
\cline { 2 - 8 } & Kontexte & Fehler & Kontexte & Fehler & Kontexte & Fehler \\
\hline Julia & 7 & 1 & 9 & 0 & 13 & 0 \\
\hline Pola & 11 & 0 & 7 & 0 & 11 & 2 \\
\hline Zusammen & 81 & 3 & 71 & 2 & 102 & 3 \\
\hline Korrektheit & \multicolumn{2}{|c|}{$96 \%$} & \multicolumn{2}{|c|}{$97 \%$} & \multicolumn{2}{c|}{$97 \%$} \\
\hline
\end{tabular}

Die Ergebnisse der Aufgabe zur schriftlichen Sprachproduktion zeigen, dass die Probanden keine Schwierigkeiten mit der korrekten Realisierung der Inversion, Verbalklammer und Verbendstellung haben. Die Korrektheit beträgt jeweils über 96 Prozent und Fehler kommen nur vereinzelt vor.

\subsection{Ergebnisse der mündlichen Übersetzungsaufgabe}

In der Auswertung der Daten aus der mündlichen Übersetzungsaufgabe wurden mehrere Teilanalysen durchgeführt. Zuerst wurden alle Sätze zusammen analysiert, um die allgemeine Korrektheit und Akkuratheit der Übersetzungen auszuwerten. Allgemeine Korrektheit bedeutet den Prozentsatz aller korrekt übersetzten Sätze, unabhängig von den untersuchten Verbstellungsmustern. Bei der Akkuratheit handelt es sich darum, ob die Probanden alle Elemente im Satz übersetzt haben oder ein Element, z. B. eine Angabe im Mittelfeld, ausgelassen haben. Die allgemeine Akkuratheit steht somit für jenen Prozentsatz der Sätze, die in ihrer Gesamtheit übersetzt wurden, ohne dass ein Element ausgelassen wurde. Abbildung 1 stellt die allgemeine Korrektheit und Akkuratheit in der Übersetzungsaufgabe dar.

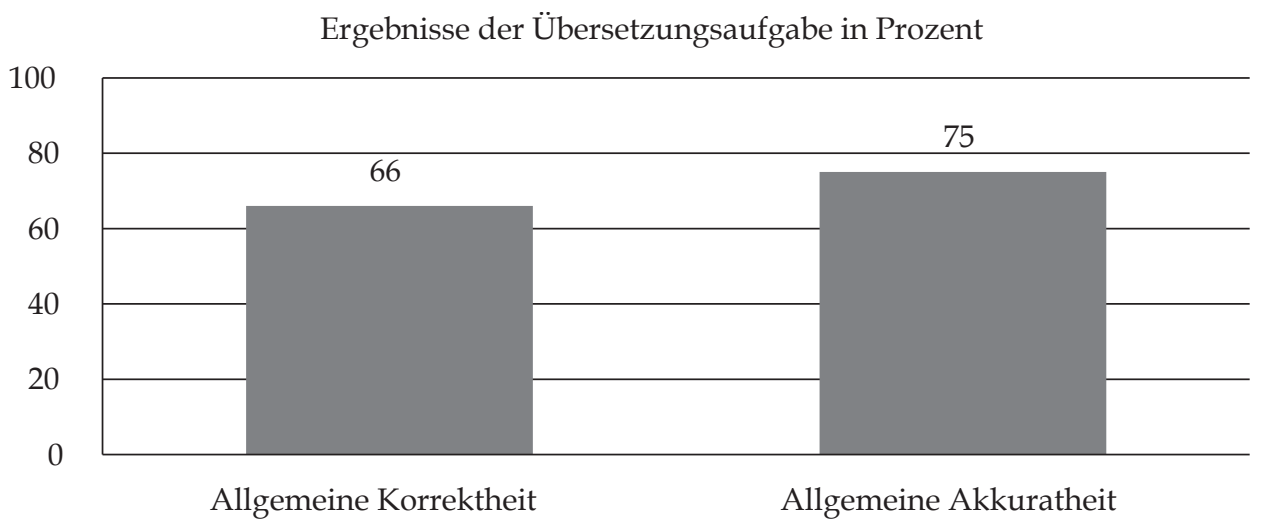

Abbildung 1. Allgemeine Korrektheit und Akkuratheit in der Übersetzungsaufgabe 
Der Abbildung kann entnommen werden, dass die allgemeine Korrektheit relativ klein ist. Außer Fehlern in der Verbstellung wurden auch elf Genusfehler, 16 Kasusfehler, fünf Deklinationsfehler und sechs Fehler im Gebrauch von Pronomen gefunden. Tabelle 4 zeigt einige Beispiele.

Tabelle 4. Beispiele für andere Fehler in den Übersetzungen

\begin{tabular}{|l|l|}
\hline \multicolumn{1}{|c|}{ Fehlertyp } & \multicolumn{1}{c|}{ Beispiele } \\
\hline Genusfehler & *Heute ist das Bus früher angekommen. \\
\hline Kasusfehler & *Ich habe nicht auf dein Bruder gewartet. \\
\hline Deklinationsfehler & *Alle wilde Tiere können leider schnell laufen. \\
\hline Fehler im Gebrauch von Pronomen & *Jan muss heute ihren Zimmer aufräumen. \\
\hline
\end{tabular}

Der Prozentsatz der allgemeinen Akkuratheit deutet darauf hin, dass die Probanden in einem Viertel aller Sätze mindestens eine Satzangabe auslassen. Getrennt berechnet wurde auch die Akkuratheit für jedes Verbstellungsmuster, welche aus Abbildung 2 abzulesen ist.

Akkuratheit in Prozent

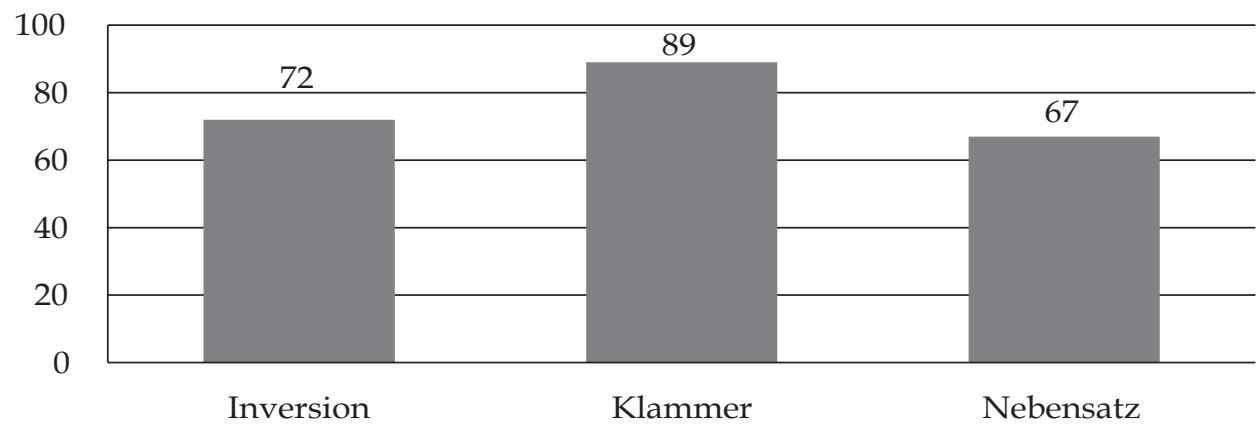

Akkuratheit Sprechen

Abbildung 2. Akkuratheit für die einzelnen Verbstellungsmuster in der Übersetzungsaufgabe

Im Vergleich zu den Sätzen mit Verbalklammer, die akkurat übersetzt werden, werden in den Sätzen mit Inversion und Verbendstellung in Nebensätzen mehr Angaben ausgelassen. Die Korrektheit für die einzelnen Phänomene zeigt, dass den Probanden die Inversion und Verbendstellung in Nebensätzen am schwersten fallen, wohingegen die Verbalklammer in 99 Prozent korrekt produziert wird. 


\section{Korrektheit in Prozent}

99

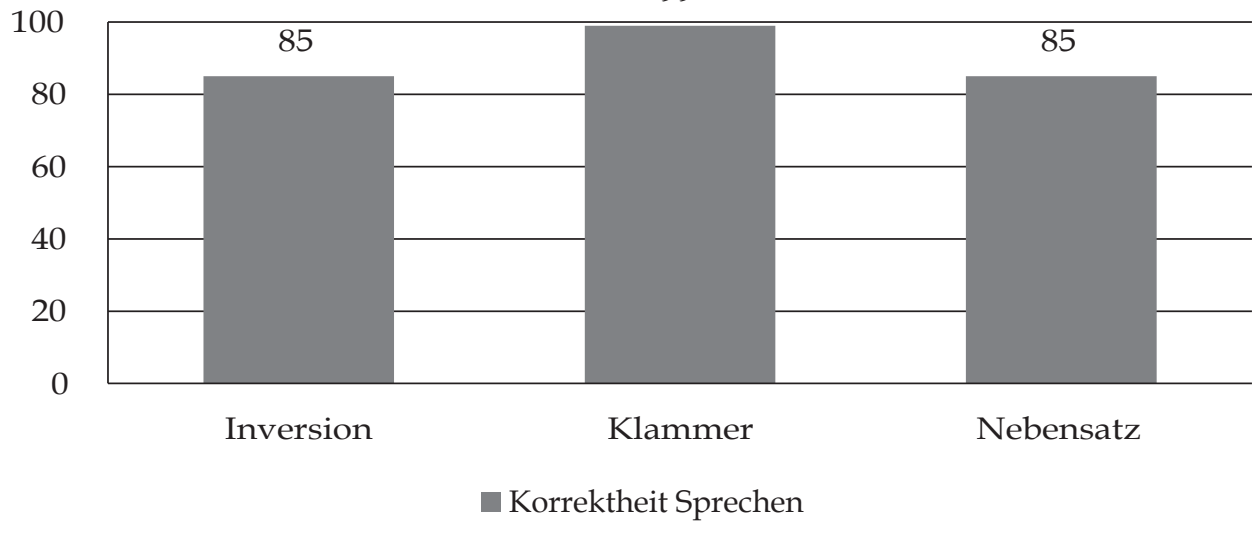

Abbildung 3. Korrektheit für die einzelnen Verbstellungsmuster in der Übersetzungsaufgabe

Der Blick auf die einzelnen Profile der Probanden in Tabelle 5 zeigt, dass die Inversion und die Verbendstellung trotz der gleichen Korrektheit im Grunde unterschiedlich realisiert werden. Während fast alle Probanden, außer Kaja, Fehler in der Inversion machen, wird die Verbendstellung sogar von vier Probanden fehlerlos angewendet. Der korrekte Gebrauch der Verbendstellung ist somit im Vergleich zu den anderen Phänomenen am instabilsten.

Tabelle 5. Korrektheit für die einzelnen Probanden in der Übersetzungsaufgabe (in \%)

\begin{tabular}{|l|c|c|c|}
\hline Proband(in) & Inversion & Verbalklammer & $\begin{array}{c}\text { Verbendstellung in } \\
\text { Nebensätzen }\end{array}$ \\
\hline Lena & 90 & 100 & 75 \\
\hline Ala & 86 & 86 & 50 \\
\hline Maks & 78 & 100 & 100 \\
\hline Kaja & 100 & 100 & 67 \\
\hline Ola & 67 & 100 & 80 \\
\hline Maja & 87 & 100 & 100 \\
\hline Anna & 90 & 100 & 67 \\
\hline Nina & 91 & 100 & 80 \\
\hline Julia & 78 & 100 & 100 \\
\hline Pola & 78 & 100 & 100 \\
\hline Mittelwert & $\mathbf{8 5}$ & $\mathbf{9 9}$ & $\mathbf{8 5}$ \\
\hline
\end{tabular}


Die Fehler in der Inversion kommen einerseits dadurch zustande, dass die Probanden die Verbzweitstellung nicht beachten, sodass sie abweichende V3-Strukturen produzieren (vgl. Beispiel 6). Andererseits stellen sie das Verb in den übergeordneten Sätzen nicht voran (vgl. Beispiel 7).

6. *Heute der Bus kommt nicht später.

7. *Wie du weißt, ich studiere seit zwei Jahren Linguistik.

Hinsichtlich der Verbendstellung ist zu bemerken, dass die Probanden keine Probleme mit Sätzen haben, in denen Konjunktionen vorkommen. Als problematisch erweist sich nur der Satz mit dem Fragepronomen "wie“ (vgl. Beispiel 8) und der temporale Nebensatz mit „wann“ (vgl. Beispiel 9).

8. *Sag mir mal, wie geht es dir.

9. *Wenn ich höre Radio, fühle ich mich in der Regel entspannt.

Eine komparative Analyse der Ergebnisse der Aufgabe zur schriftlichen Produktion und der Übersetzungsaufgabe demonstriert, dass der Unterschied zwischen den beiden Produktionsmodi relativ groß ist, aber nur für die Inversion und die Verbendstellung in Nebensätzen.

Korrektheit in Prozent

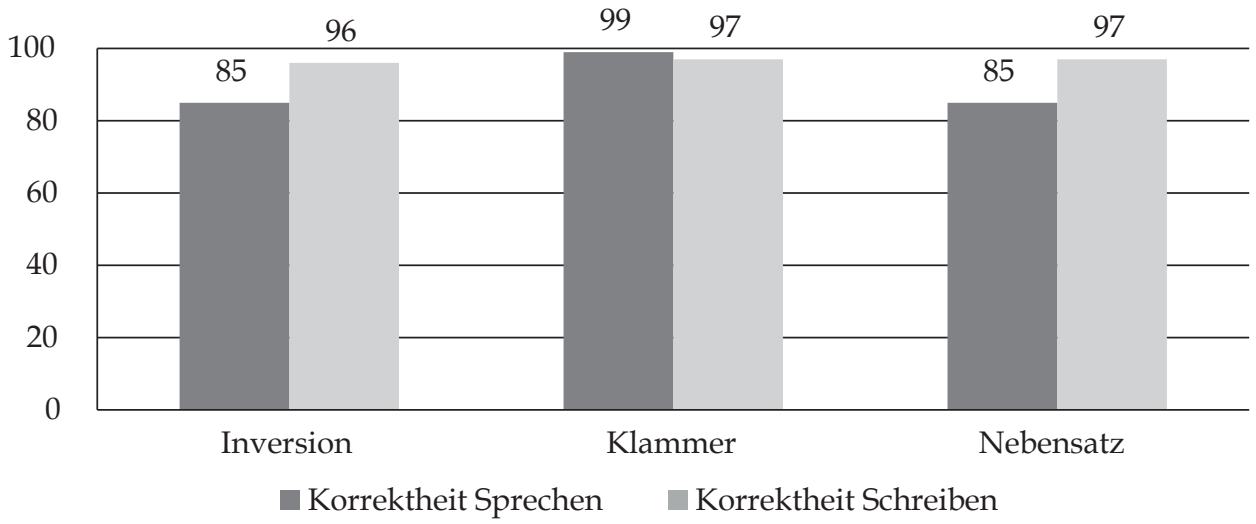

Abbildung 4. Vergleich der Korrektheit in der Aufgabe zur schriftlichen Produktion und in der Übersetzungsaufgabe

Beim Schreiben begehen die Probanden fast keine Fehler in der Wortstellung. Die Diskrepanz zwischen den Aufgaben macht sich nur bei Sätzen mit Inversion und Verbendstellung bemerkbar. 


\section{DISKUSSION}

Die Ergebnisse der Studie erlauben es, viele informative Schlussfolgerungen zu ziehen. Zuerst werden diese in Bezug auf die vier gestellten Forschungsfragen diskutiert.

1) Die Ergebnisse zeigen, dass nicht alle Verbstellungsmuster auf gleichem Niveau beherrscht werden. Die Differenzen im Gebrauch der einzelnen Verbpositionen tauchen in der schriftlichen Sprachproduktion nicht auf und machen sich erst in der mündlichen Sprachproduktion bemerkbar. Während die Probanden keine Probleme mit dem korrekten Einsatz der Verbalklammer haben, scheinen Inversion und Verbendstellung in Nebensätzen für sie viel schwieriger zu sein. Bei genauerem Hinsehen bringen die einzelnen Profile der Probanden einen Unterschied zwischen dem Beherrschen der Inversion und der Verbendstellung ans Licht, obwohl sie sich in der Gesamtdarstellung der Korrektheit ebenbürtig sind. Hundertprozentige Korrektheit wird nämlich nur von einem Probanden für die Inversion, aber bereits von vier Probanden für die Verbendstellung erreicht. Die Ergebnisse zeigen aber gleichzeitig auch, dass vier Probanden eine höhere Korrektheit für die Inversion als für die Verbendstellung erzielen. Es lässt sich somit nicht eindeutig entscheiden, welches der zwei Verbstellungsmuster tatsächlich problematischer ist. Die Differenzen zwischen den einzelnen Phänomenen sind außerdem in der Akkuratheit erkennbar. Darüber hinaus deutet die niedrige Akkuratheit auf Sprachverarbeitungsprobleme beim Übersetzen der Sätze mit Inversion und Verbendstellung in Nebensätzen hin. Die Probanden lassen dabei oft lokale, temporale und modale Angaben im Mittelfeld aus, weil sie infolge eines erhöhten kognitiven Aufwandes nicht in der Lage sind, alle Informationen parallel zu integrieren. Möglicherweise ergeben sich die Probleme bei der Verarbeitung der Verbendstellung in Nebensätzen nicht aus der Überbelastung des Arbeitsgedächtnisses, weil die Verbalklammer, die auch das temporäre Beibehalten der aktivierten Repräsentation eines Verbs erfordert, in Hinsicht auf die Akkuratheit nicht diffizil erscheint. Inversion und Verbendstellung sind für die Probanden schwieriger und nehmen daher mehr kognitive Ressourcen in Anspruch. Dadurch werden andere Aspekte, die beim Übersetzen zu berücksichtigen wären, benachteiligt.

2) Der Befund, dass die Probanden die Verbalklammer vor der Inversion und Verbendstellung erworben haben, unterstützt die Hypothese der natürlichen Erwerbssequenz nur bedingt. Angesichts der oben diskutierten interindividuellen Variationen im Gebrauch der Inversion einerseits und der Verbendstellung andererseits, kann die Reihenfolge ihres Erwerbs nicht ermittelt werden. Allerdings suggerieren die Ergebnisse der schriftlichen Aufgabe, dass das Wissen um die 
untersuchten Verbstellungsmuster schon vorhanden ist. Offensichtlich kann es aber bei der mündlichen Produktion nicht völlig korrekt umgesetzt werden.

3) Wie Riehl (2018: 286) zu Recht anmerkt, steht bei der schriftlichen Sprachproduktion mehr Planungszeit zur Verfügung, weshalb der Prozess des Monitorings viel stärker ausgeprägt ist. Beim Schreiben haben die Lerner auch Zeit, auf das explizite Regelwissen sowie auf das metasprachliche Wissen zuzugreifen, wodurch weniger grammatische Fehler begangen werden. Wenn die Probanden weniger Zeit für die Übersetzung hätten, würden sie möglicherweise auch mehr Fehler in der Verbstellung machen. Unter Zeitdruck wäre nämlich der Zugang zum expliziten Grammatikwissen wesentlich eingeschränkt, wodurch die Probanden größtenteils auf das implizite Grammatikwissen angewiesen wären. Die Diskussion um das Verhältnis zwischen dem impliziten und expliziten Grammatikwissen im unterrichtlichen Fremdsprachenerwerb ist enorm komplex und kann in diesem Rahmen leider nicht beleuchtet werden. ${ }^{5}$

4) Laut den Ergebnissen der vorliegenden Untersuchung ist eher davon auszugehen, dass der syntaktische Transfer aus der Erstsprache Polnisch und der Zweitsprache Englisch keine bedeutsame Rolle beim untersuchten Erwerb der Verbstellung im Deutschen als Fremdsprache spielt. Eine Bestätigung des syntaktischen Transfers würde in den untersuchten Bereichen darin bestehen, dass die Probanden die für das Polnische und Englische charakteristischen Wortstellungsmuster sowohl in der schriftlichen als auch in der mündlichen Sprachproduktion bevorzugen. ${ }^{6}$ Dies ist eindeutig nicht der Fall. Darüber hinaus sind strukturelle Unterschiede zwischen den involvierten Sprachen ähnlich für alle drei Verbstellungsmuster. Daher gibt es keinen ersichtlichen Grund, warum der Transfer nur ein Phänomen, z. B. die Inversion, betreffen sollte.

Die Analyse der mündlichen Daten weist ferner darauf hin, dass Kognaten keinen Einfluss auf die Korrektheit und Akkuratheit in der Übersetzungsaufgabe haben. Was das Code-Switching betrifft, so kommt es nur in vier übersetzten Sätzen vor, und zwar unabhängig davon, ob sie die Kognaten involvieren oder nicht:

10. Wilde Tiere können leider fast laufen.

11. Morgen after der Schule muss ich mit meiner Mutter sprechen.

In seiner Triggering-Hypothese spricht Clyne (1991: 193-195) von den sogenannten Auslösewörtern (trigger words), die ein Code-Switching induzieren können. Hierzu zählen u. a. Kognaten, die eine Schnittstelle zwischen den mentalen Lexika

\footnotetext{
${ }^{5}$ Vgl. Sadownik (2006) für eine ausführliche Auseinandersetzung mit diesem Problembereich.

${ }^{6}$ Vgl. Hinc (2010) für eine Diskussion über Transferpotenziale beim Erwerb der Wortstellung im Deutschen als Fremdsprache bei polnischen Lernern mit Zweitsprache Englisch.
} 
zweier oder mehrerer Sprachen bilden. Die Ergebnisse der vorliegenden Studie legen nahe, dass der Prozess des Triggering beim Übersetzen kaum erfolgt, zumindest bei der gegebenen Zeit für die Ausführung der Aufgabe. Das rührt wahrscheinlich daher, dass das Übersetzen unter experimentellen Bedingungen im Vergleich zur freien Sprachproduktion weniger natürlich erscheint und mit der kognitiven Kontrolle zusammenhängt, die die nicht-intendierte Sprache aktiv inhibiert.

\section{FAZIT}

Die vorliegende Studie hatte zum Ziel, die Entwicklung der grundlegenden Verbstellungsmuster in einem fortgeschrittenen Erwerbsstadium des Deutschen als Fremdsprache zu untersuchen. Während die polnischen DaF-Lerner keine Schwierigkeiten mit der Verbalklammer hatten, erwiesen sich die Inversion und Verbendstellung in Nebensätzen als problematisch. Dieser Befund entspricht der natürlichen Erwerbssequenz, die bereits in vielen Studien zum natürlichen wie auch gesteuerten Grammatikerwerb ermittelt wurde. Die parallele Analyse der schriftlichen und mündlichen Sprachproduktion brachte Unterschiede zwischen diesen beiden Sprachmodi zum Vorschein. Die sehr hohe Korrektheit in der schriftlichen Aufgabe deutete darauf hin, dass die Regeln der Verbstellung schon erworben wurden. Allerdings begangen die Lerner mehr Fehler in der mündlichen Übersetzungsaufgabe, aber nur in Bezug auf die Inversion und die Verbendstellung in Nebensätzen. Die Diskrepanz zwischen der schriftlichen und der mündlichen Sprachproduktion wurde als Evidenz für Verarbeitungsprobleme bei der Übersetzung der Sätze mit den zwei problematischen Verbstellungsmustern interpretiert. Resümierend ist festzuhalten, dass Inversion und Verbendstellung in Nebensätzen Erwerbshürden darstellen, die sogar für sehr fortgeschrittene DaF-Lerner schwer zu überwinden sind.

\section{LITERATURVERZEICHNIS}

Ballestracci, S. (2007). Zum DaF-Erwerb ausgewählter grammatischer Strukturen der deutschen Sprache durch italophone Studierende. Ergebnisse und didaktische Konsequenzen. Studi Linguistici e Filologici Online. Rivista Telematica del Dipartimento di Linguistica dell'Università di Pisa (SLiFO), 5 (1), 1-50.

Barczyk, S. (2008). Entwicklungsprofile im Syntaxerwerb des Deutschen durch polnische Lerner psycholinguistische und glottodidaktische Aspekte. In: K. Pittner (Hrsg.), Beiträge zu Sprache und Sprachen 6. Vorträge der 16. Jahrestagung der Gesellschaft für Sprache und Sprachen (Gesus e.V.) (S. 377-388). München: Lincom Europa.

Clahsen, H. / Meisel, J.M. / Pienemann, M. (1983). Deutsch als Zweitsprache. Der Spracherwerb ausländischer Arbeiter. Tübingen: Narr. 
Clahsen, H. / Muysken, P. (1986). The availability of universal grammar to adult and child learners - a study of the acquisition of German word order. Second Language Research, 2 (2), 93-119.

Clyne, M. (1991). Community languages: The Australian experience. Cambridge: Cambridge University Press.

Diehl, E. / Christen, H. / Leuenberger, S. / Pelvat, I. / Studer, T. (2000). Grammatikunterricht: Alles für der Katz? Untersuchungen zum Zweitspracherwerb Deutsch. Tübingen: Max Niemeyer Verlag.

Dijkstra, T. (2009). The multilingual lexicon. In: G.M. Gaskell (Hrsg.), The Oxford handbook of psycholinguistics (S. 251-265). Oxford: Oxford University Press.

Długosz, K. (2018). Transfererscheinungen zu Beginn des L3-Erwerbs: Evidenz aus der schriftlichen Sprachproduktion mehrsprachiger Deutschlerner. Kwartalnik Neofilologiczny, LXV (4), 632-647. DOI: $10.24425 / \mathrm{kn} .2018 .125009$.

Edmondson, W.J. / House, J. (2006). Einführung in die Sprachlehrforschung. Tübingen: Narr.

Ellis, R. (1989). Are classroom and naturalistic acquisition the same? A study of the classroom acquisition of German word order rules. Studies in Second Language Acquisition, 11, 305-328. DOI: $10.1017 /$ S0272263100008159.

Engel, U. / Rytel-Kuc, D. / Cirko, L. / Dębski, A. / Gaca, A. / Jurasz, A. / Kątny, A. / Mecner, P. / Prokop, I. / Sadziński, R. / Schatte, Ch. / Schatte, C. / Tomiczek, E. / Weiss, D. (2000). Deutsch-polnische kontrastive Grammatik. Band 1. Warszawa: Wydawnictwo Naukowe PWN.

Hinc, J. (2010). Englisch als Interferenzquelle bei der Aneignung der Wortstellung des Deutschen: Sprachvergleichende Bemerkungen aus der Sicht des Polnischen als Muttersprache des Englischen als erster Fremdsprache und des Deutschen als zweiter Fremdsprache. Glottodidactica, XXXVI, 143-154.

Meisel, J.M. / Clahsen, H. / Pienemann, M. (1981). On determining developmental stages in natural second language acquisition. Studies in Second Language Acquisition, 3, 109-135. DOI: 10.1017/ S0272263100004137.

Odendahl, W. (2016). Blattdolmetschen als Möglichkeit zur Integration von Dolmetschertraining in den Mittelstufenunterricht. Info DaF, 1, 21-43. DOI: 10.1515/infodaf-2016-0104.

Pfeiffer, W. (2001). Glottodidaktik als Wissenschaft. In: K. Aguado / C. Riemer (Hrsg.), Wege und Ziele. Zur Theorie, Empirie und Praxis des Deutschen als Fremdsprache (und anderer Fremdsprachen). Festschrift für Gert Henrici zum 60. Geburtstag (S. 63-96). Hohengehren: Schneider Verlag.

Riehl, C.M. (2018). Analyse schriftlicher Lernervarietäten. In: J. Roche / E. Terrasi-Haufe (Hrsg.), Mehrsprachigkeit und Sprachenerwerb (S. 285-299). Tübingen: Narr.

Sadownik, B. (1997). Glottodidaktische und psycholinguistische Aspekte des Fremdsprachenerwerbs: Lernerperspektive. Lublin: Wydawnictwo UMCS.

Sadownik, B. (2006). Zur Relation zwischen explizitem und implizitem Grammatikwissen im unterrichtlichen Fremdsprachenerwerb. Lublin Studies in Modern Languages and Literature, 29/30, 193-217.

Sadownik, B. (2008). The acquisition of German syntax by Polish learners in classroom conditions. In: D. Gabryś-Barker (Hrsg.), Morphosyntactic issues in second language acquisition (S. 203-224). Clevedon / Buffalo / Toronto: Multilingual Matters.

Sopata, A. (2004). Universalgrammatik und Fremdsprachendidaktik. Frankfurt a. M.: Peter Lang Verlag. Sopata, A. (2018). Entwicklung der Informationsstruktur beim Erwerb des Deutschen als frühe Fremdsprache. Glottodidactica, XLV (2), 285-298. DOI: 10.14746/g1.2018.45.2.16.

Sopata, A. / Długosz, K. (2020). Deutsch als schwächere Sprache bei deutsch-polnisch bilingualen Kindern: Studie zur Negation- und Verbstellung. Zeitschrift für Germanistische Linguistik, 48 (2), 269-297. DOI: 10.1515/zgl-2020-2003. 
Terrasi-Haufe, E. (2004). Der Schulerwerb von Deutsch als Fremdsprache. Eine Empirische Untersuchung am Beispiel der italienischsprachigen Schweiz. Tübingen: Niemeyer.

Tschirner, E. (1999). Lernergrammatiken und Grammatikprogression. In: B. Skibitzki / B. Wojtak (Hrsg.), Linguistik und Deutsch als Fremdsprache. Festschrift für Gerhard Helbig (S. 227-240). Tübingen: Niemeyer.

Zuzok, N. (2010). Interkulturelle Kompetenz im Fremdsprachenunterricht Deutsch nach Englisch: Eine Pilotuntersuchung der Klassen 4-6 im Raum Poznań. Glottodidactica, XXXVI, 235-243.

Received: 02.07.2019; revised: 21.04 .2020

\section{KAMIL DŁUGOSZ}

Uniwersytet im. Adama Mickiewicza w Poznaniu

kamil.dlugosz@amu.edu.pl

ORCID: 0000-0002-9600-6452 
